\title{
Pausas activas para el control de trastornos músculo- esqueléticos en los odontólogos del seguro social campesino de la provincia de Cotopaxi
}

Dennis Hedenia Pilco Albán. ${ }^{1}$, Manolo Alexander Córdova Suarez. ${ }^{2}$ \& Edison Patricio Villacres Cevallos. ${ }^{3}$

Implementation of active pauses for the control of musculoskeletal disorders in the dentists of the rural social security of the province of Cotopaxi

Recibido: 03-08-2021 / Revisado: 19-08-2021 /Aceptado: 23-08-2021/ Publicado: 05-09-2021

\section{DOI: 10.33262/anatomiadigital.v4i3.1.1898}

\begin{abstract}
Introduction. The activities that dentists develop in the Peasant Social Insurance generate damage to their health and early manifestations of musculoskeletal disorders are continually presented by adopting static postures and executing constant repetitive movements. Objective. The effect of the implementation of a program of active pauses for the control of musculoskeletal disorders generated by repetitive movements in the dentists of the Rural Social Security of Cotopaxi was tested. Methodology. A plan of active breaks was developed, which was then socialized with

\section{Resumen}

Introducción. Las actividades que los odontólogos desarrollan en los Seguros Sociales Campesinos generan daños en su salud y continuamente se presentan manifestaciones tempranas de trastornos musculoesqueléticos al adoptar posturas estáticas y ejecutar movimientos repetitivos constantes. Objetivo. Se probó el efecto de la implementación de un programa de pausas activas para el control de trastornos músculo esqueléticos generados por movimientos repetitivos en los odontólogos del Seguro Social Campesino de Cotopaxi. Metodología. Se

\footnotetext{
1 Universidad Regional Autónoma de los Andes, Posgradista. Ambato-Ecuador, pg.dennishpa04@uniandes.edu.ec ORCID: 0 (iD000-0001-6272-0992

2 Universidad Nacional de Chimborazo, Facultad de Ingeniería, Riobamba-Ecuador, manolo.cordova@unach.edu.ec ORCID: (iD0000-0001-6786-7926

3 Universidad Nacional de Chimborazo, Facultad de Ingeniería, Riobamba-Ecuador, pvillacres@ unach.edu.ecORCID: (D) 00000001-9518-1278
} 
the exposed personnel for its execution. Finally, the efficacy of the active breaks program was analyzed by applying the Occupational Repetitive Action (OCRA) method before and after the intervention in the recovery factor, leaving a period of action of two weeks. Results. The implementation of 2 sessions of active breaks of ten minutes a day each, decreased the value of the Ocra index by $74 \%$, giving the following results of the factors: with the following considerations: Help from an assistant and improvement of the manual tool by an automatic one. Conclusion. By implementing active breaks and providing the help of an assistant, the level of ergonomic risk due to repetitive movement was reduced by $70,05 \%$ due to the OCRA index, going from intolerable to medium.

Keywords: ergonomic, dentists, OCRA active pauses . empezó desarrollando un plan de pausas activas que luego se socializó al personal expuesto para su ejecución. Finalmente se analizó la eficacia del programa de pausas activas aplicando el método Occupational Repetitive Action (OCRA) antes y después de la intervención en el factor de recuperación dejando un lapso de actuación de dos semanas. Resultados. La implementación de 2 sesiones de pausas activas de diez minutos al día cada una, disminuyeron el valor del índice Ocra un $74 \%$ dando los siguientes resultados de los factores: con las siguientes consideraciones: Ayuda de un asistente y mejora de la herramienta manual por una automática. Conclusión. Al implementar pausas activas y dotar de ayuda de un asistente se redujo un 70,05\% el nivel de riesgo ergonómico por movimiento repetitivo por el índice OCRA pasando de intolerable a medio.

Palabras claves: ergonómico, odontólogos, OCRA pausas activas.

\section{Introducción}

Es bien conocido que los factores de riegos ergonómicos se manifiestan como una problemática que se adquiere en algunos centros de salud del país, en donde la demanda de la jornada laboral es elevada y el trabajador va desvalorizando su salud, trayendo como consecuencia repercusiones a nivel físico y psicológico, en primera instancia (Laguerre, 2019). En el Ecuador el riesgo ergonómico no es la prioridad de los servicios de salud pública, puesto que pese a que son organismos de control las unidades de seguridad y salud en el trabajo no están constituidas en su totalidad y la gestión de la prevención se la encarga a personal con desconocimiento y a veces hasta improvisado (Martínez Barranco \& Yandún Burbano, 2017).

En Odontología la ergonomía es muy importante ya que se ve inmiscuido con muchas posturas que el profesional adquiere en el momento de realizar su trabajo práctico, el cual va a depender de diferentes factores entre ellos está el diseño de puesto de trabajo, las 
dimensiones antropométricas del cuerpo trabajador y las dimensiones del paciente. Posteriormente si no se toma en cuenta las posturas adecuadas al pasar del tiempo estas pueden desencadenar en distintos trastornos musculo esqueléticos (Buitrón Carrera, 2015).

\section{Marco Teórico}

Una de las alternativas para el control de los riesgos ergonómicos son la adopción de pausas activas, ya que ayuda al factor de recuperación del individuo y permite soportar la fatiga laboral (Ribadeneira Campozano, 2015).

Los programas de pausas activas en el trabajo, incluyen una rutina que generalmente incorpora movimientos articulares, de estiramiento de los diferentes grupos musculares, como son cabeza y cuello, hombros, codos, manos, tronco, piernas y pies; otra técnica que se suele utilizar son ejercicios de respiración, para activar la circulación sanguínea y la energía corporal previniendo problemas causados por la fatiga física y mental a la vez que favorece el funcionamiento cerebral y con ello incrementa la productividad (Niño Peña \& Solano Aguado, 2020), no obstante lo anterior, es deseable incorporar actividades que involucren interacción entre compañeros, como juegos, bailes de parejas y grupales que de acuerdo a la experiencia mejoran el clima y las relaciones dentro del área laboral (Romero Moreno \& Córdoba Quejada, 2021).

Los beneficios de la actividad física regular son conocidos y considerados como uno de los componentes más importantes de un estilo de vida saludable por que se vincula a beneficios físicos y mentales (Corbí-Santamaría et al., 2014). Realizar al menos 30 minutos diarios de actividad física, dicha actividad aporta beneficios a la salud y basta con que un adulto acumule30 minutos diarios de intensidad moderada para lograr beneficios visibles (Cordero et al., 2014).

Estas recomendaciones son factibles de desarrollarlas en el lugar de trabajo y sin tener que cambiar de vestuario puesto que se refieren a acciones de la vida cotidiana; la actividad física moderada pero regular, subir escaleras, caminar rápidamente y montar en bicicleta como parte de la rutina diaria, puede reducir el estrés, aliviar la depresión y la ansiedad, estimular la autoestima y mejorar la claridad mental.

Por otro lado, los odontólogos ejecutan la mayor de sus actividades realizando movimientos repetitivos y los indicadores de enfermedades ocupacionales hacen de esta actividad una de las más peligrosa para generar molestias relacionadas a daños en músculos, tendones y huesos. Los servicios de salud y sobre todo los del sector público en el Ecuador no son la excepción, puesto a que la carencia de programas de control por falta de la gestión de la prevención deja a los trabajadores de este sector vulnerables a molestias, manifestaciones tempranas, y hasta accidentes. Para establecer el cambio por la implementación de medidas 
de control ergonómico es necesario utilizar alguna herramienta que ayude a establecer una línea base cuali cuantitativa. De los métodos más utilizados se reconoce al que menciona la norma UNE EN 1005-5 como adecuado el check-list occupational repetitive action (OCRA) (Rosero Menéndez, 2012). Es decir evalúa la repetitividad de las tareas que provocan repeticiones en el mismo ciclo de trabajo, acciones técnicas y movimientos (Cimatti et al., 2013).

Esta investigación establece la mejora al cambio ocasionada por la implantación de un programa de pausas activas evidenciada por el método OCRA.

\section{Metodología}

La modalidad adoptada para la presente investigación, es el de tipo cuali-cuantitativo, el aspecto cualitativo se lo aplicó en base a la observación de las posturas inadecuadas que tienen los profesionales odontólogos de la provincia de Cotopaxi. En cuanto a lo cuantitativo, se lo hizo en base a una hoja de cálculo con el método OCRA.

\section{Aplicación del Método OCRA}

El método OCRA servirá para determinar si el nivel de exigencias físicas impuestas por la tarea y el entorno donde aquella se desarrolla están dentro de los límites fisiológicos y biomecánicos aceptables o, por el contrario, pueden llegar a sobrepasar las capacidades físicas de la persona con el consiguiente riesgo para su salud (Soto \& Casado, 2006). Es un método de evaluación de la exposición a movimientos y esfuerzos repetitivos de los miembros superiores que permite valorar el riesgo asociado al trabajo repetitivo y da nivel de riesgo en función de la probabilidad de aparición de trastornos musculoesqueléticos en un determinado tiempo (Picazo \& de Miguel, 2003).

Una característica importante del método OCRA es su sencillez y rapidez de aplicación. La evaluación de un puesto con un ciclo de trabajo de unos 15 segundos puede realizarse hasta en 4 minutos. Para un ciclo de 15 minutos, el tiempo de evaluación puede aproximarse a 30 minutos incluyendo tareas adicionales de registro de la información.

El valor del Índice Check List OCRA (ICKL) se calcula empleando la ecuación 1:

Donde

$$
\mathrm{ICKL}=(\mathrm{FT}+\mathrm{FR}+\mathrm{FFr}+\mathrm{FP}+\mathrm{FF}) \cdot \mathrm{D} \quad(\text { Ec. } 1)
$$

ICKL = índice OCRA

FT= Factor de tiempo

$\mathrm{FR}=$ Factor de recuperación

FFr=Factor de frecuencia 
$\mathrm{FP}=$ Factor de postura

$\mathrm{FF}=$ Factor de fuerza

$\mathrm{D}=$ factor de duración

El valor de ICKL es el resultado de la suma de cinco de factores, posteriormente multiplicados por el multiplicador de duración. Como paso previo al cálculo de cada factor y del multiplicador de duración, es necesario conocer, a partir de los datos organizativos del trabajo, el tiempo neto de trabajo repetitivo y el tiempo neto de ciclo de trabajo (Işık \& Adalı, 2016).

La existencia de periodos de recuperación adecuados tras un periodo de actividad permite la recuperación de los tejidos óseos y musculares. Si no existe suficiente tiempo de recuperación tras la actividad aumenta el riesgo de padecer trastornos de tipo musculoesquelético. Este factor de la ecuación de cálculo del índice check list OCRA valora si los periodos de recuperación en el puesto evaluado son suficientes y están convenientemente distribuidos (Grieco, 1998). Para determinar el valor del factor frecuencia es necesario identificar el tipo de las acciones técnicas realizadas en el puesto. Se distinguen dos tipos de acciones técnicas: estáticas y dinámicas. Las acciones técnicas dinámicas se caracterizan por ser breves y repetidas (Alvaro \& Ricardo, 2017). Las acciones técnicas estáticas se caracterizan por tener una mayor duración y se deben analizarse por separado. Además, se analizarán por separado las acciones realizadas por ambos brazos, debiendo realizar una evaluación diferente para cada brazo si es necesario.

\section{Resultados y Discusión}

En la tabla 1. Se observan los resultados iniciales del puesto de trabajo de odontólogo.

\section{Tabla 1.}

Evaluación con el Método OCRA

\section{Factores repetitivos}

Organización del tiempo de trabajo

\begin{tabular}{|c|c|}
\hline Tiempo de exposición & 8 horas \\
\hline $\begin{array}{l}\text { Pausas y tareas no repetitivas (pausas } \\
\text { oficiales) }\end{array}$ & 0,25 horas \\
\hline $\begin{array}{l}\text { Pausas y tareas no repetitivas (pausas } \\
\text { no oficiales) }\end{array}$ & 0 horas \\
\hline $\begin{array}{l}\text { Duración del descanso para el } \\
\text { almuerzo }\end{array}$ & 0,5 horas \\
\hline Duración de tareas no repetitivas & 0 minutos \\
\hline \multicolumn{2}{|c|}{ Periodos de recuperación } \\
\hline Periodos de recuperación & $\begin{array}{l}\text { No hay pausas reales excepto por unos minutos en un } \\
\text { turno de } 7 \text { a } 8 \text { horas }\end{array}$ \\
\hline
\end{tabular}




\section{Tabla 1.}

Evaluación con el Método OCRA

(continuación)

\begin{tabular}{|c|c|}
\hline \multicolumn{2}{|c|}{ Frecuencias y tipos de acciones } \\
\hline Tiempo del ciclo & 360 segundos \\
\hline Número de acciones Técnicas & 6 \\
\hline Tipo de Acciones & Dinámicas y estáticas \\
\hline Acciones Técnicas Estáticas & $\begin{array}{l}\text { Se sostiene un objeto al menos } 5 \text { segundos consecutivos, } \\
\text { durante } 3 / 3 \text { del tiempo de ciclo }\end{array}$ \\
\hline Acciones Técnicas Dinámicas & $\begin{array}{l}\text { Movimientos del brazo bastante rápidos (más de } 40 \\
\text { acciones/minuto). Con pequeñas pausas ocasionales. }\end{array}$ \\
\hline \multicolumn{2}{|r|}{ Posturas adoptadas } \\
\hline Hombro & El brazo no posee apoyo más de la mitad del tiempo. \\
\hline Codo & Movimientos repentinos casi todo el tiempo \\
\hline Muñeca & $\begin{array}{l}\text { Permanece doblada en una posición extrema todo el } \\
\text { tiempo. }\end{array}$ \\
\hline Mano & Los dedos están en forma de gancho. Todo el tiempo. \\
\hline Movimientos estereotipados & $\begin{array}{l}\text { Repetición de acciones con movimientos iguales del } \\
\text { hombro, codo, muñeca, o dedos casi todo el tiempo }\end{array}$ \\
\hline \multicolumn{2}{|r|}{ Fuerza } \\
\hline \multirow[t]{2}{*}{ Actividades que implican esfuerzo } & $\begin{array}{l}\text { Cerrar o abrir, con fuerza intensa más del } 10 \% \text { del } \\
\text { tiempo }\end{array}$ \\
\hline & $\begin{array}{l}\text { Se usa herramientas, con fuerza moderada por } 1 / 3 \text { del } \\
\text { tiempo }\end{array}$ \\
\hline \multicolumn{2}{|l|}{ Factores de riesgo adicionales } \\
\hline Tarea de precisión & Mas de la mitad del tiempo. \\
\hline Ritmo de trabajo & Ritmo totalmente determinado por la máquina \\
\hline \multicolumn{2}{|r|}{ Resultado final OCRA } \\
\hline \multicolumn{2}{|r|}{70,5} \\
\hline \multicolumn{2}{|r|}{ Resultado } \\
\hline & esgo inaceptable Alto \\
\hline
\end{tabular}

Nota: Los datos ingresados son el resultado de una pre - investigación de dos semanas.

Para el programa de pausas activas se capacitaron a monitores (enfermeras) que pertenecían a la misma unidad de prestación de servicios del Hospital. Supervisados por el equipo investigador se aplicaron actividades como: estiramientos, baile entretenido y ejercicios de respiración y relajación que incluía 10 min de música, respetando la parte inicial, desarrollo y final de la sesión. Esta intervención se desarrolló durante dos semanas, cinco veces a la semana por 10 min cada vez, en horarios de mañana o tarde de acuerdo con el servicio. Ver figura 1 . 


\section{ADDigital}

ISSN: 2697-3391

Figura 1.

Ejercicios de calentamiento previo actividad lúdica en pausas activas

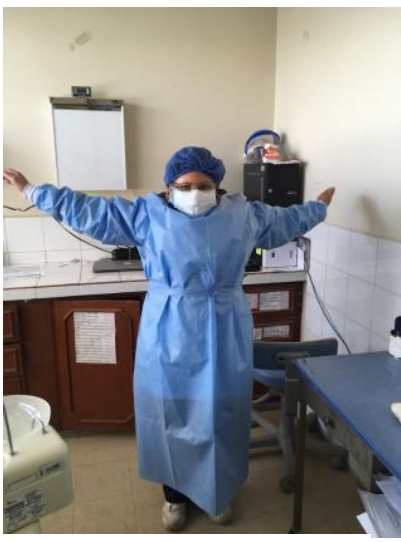

Ejerciciol.

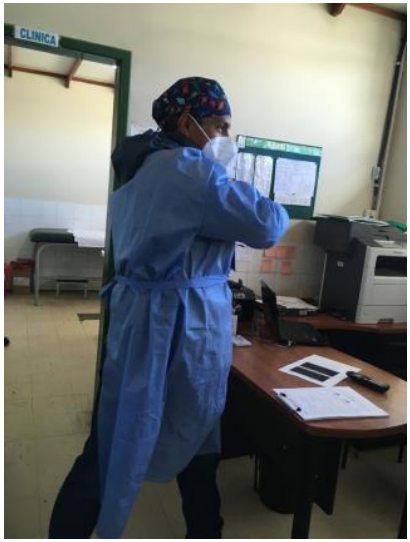

Ejercicio 4.

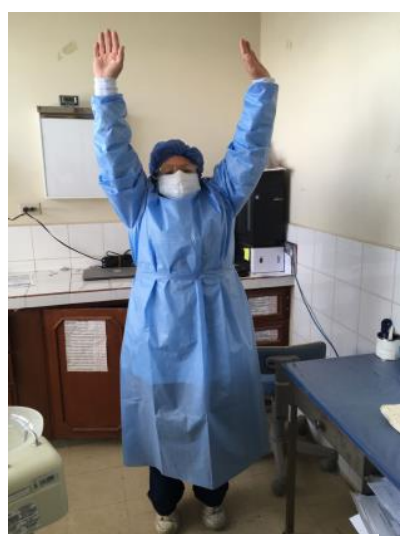

Ejercicio2.

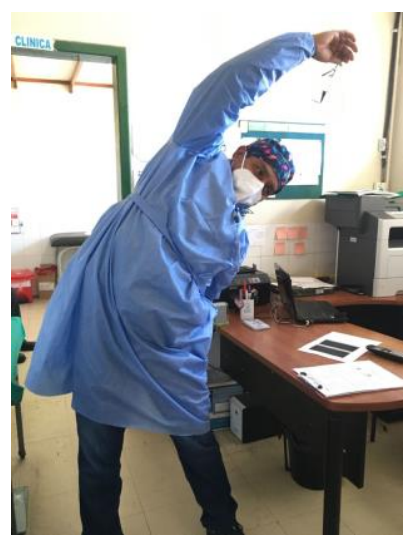

Ejercicio 5.

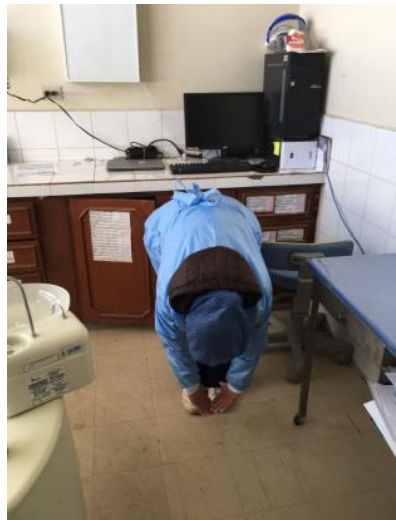

Ejercicio 3.

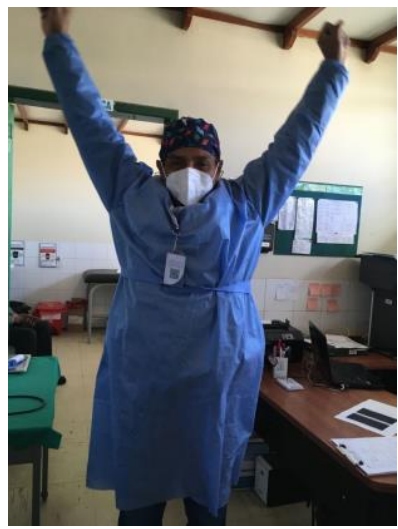

Ejercicio 6.

Luego de la intervención mediante pausas activas, los resultados obtenidos respondieron a la inquietud fundacional sobre su incidencia en la actividad física de la muestra, puesto que el Índice OCRA disminuyó notoriamente, como lo demuestra la Tabla 2. 


\section{Tabla 2.}

Evaluación OCRA luego de la intervención de pausas activas

\begin{tabular}{|c|c|}
\hline \multicolumn{2}{|c|}{ Factores repetitivos } \\
\hline \multicolumn{2}{|c|}{ Organización del tiempo de trabajo } \\
\hline Tiempo de exposición & 4 horas \\
\hline $\begin{array}{l}\text { Pausas y tareas no repetitivas } \\
\text { (pausas oficiales) }\end{array}$ & 0,66 horas \\
\hline $\begin{array}{l}\text { Pausas y tareas no repetitivas } \\
\text { (pausas no oficiales) }\end{array}$ & 0,25 horas \\
\hline $\begin{array}{l}\text { Duración del descanso para el } \\
\text { almuerzo }\end{array}$ & 0,5 horas \\
\hline Duración de tareas no repetitivas & 0 minutos \\
\hline \multicolumn{2}{|c|}{ Periodos de recuperación } \\
\hline Periodos de recuperación & Dos pausas por la mañana y dos por la tarde. \\
\hline \multicolumn{2}{|c|}{ Frecuencias y tipos de acciones } \\
\hline Tiempo del ciclo & 360 segundos \\
\hline Número de acciones Técnicas & 3 \\
\hline Tipo de Acciones & Dinámicas y estáticas \\
\hline Acciones Técnicas Estáticas & $\begin{array}{l}\text { Se sostiene un objeto al menos } 5 \text { segundos } \\
\text { consecutivos, durante } 2 / 3 \text { del tiempo de ciclo }\end{array}$ \\
\hline Acciones Técnicas Dinámicas & Movimientos del brazo no son demasiado rápidos. \\
\hline \multicolumn{2}{|c|}{ Posturas adoptadas } \\
\hline Hombro & $\begin{array}{l}\text { El brazo no posee apoyo más de la mitad del } \\
\text { tiempo. }\end{array}$ \\
\hline Codo & Movimientos repentinos casi todo el tiempo \\
\hline Muñeca & $\begin{array}{l}\text { Permanece doblada en una posición extrema todo } \\
\text { el tiempo. }\end{array}$ \\
\hline Mano & No se realizan agarres. \\
\hline Movimientos estereotipados & $\begin{array}{l}\text { Repetición de acciones con movimientos iguales } \\
\text { del hombro, codo, muñeca, o dedos casi todo el } \\
\text { tiempo }\end{array}$ \\
\hline \multicolumn{2}{|r|}{ Fuerza } \\
\hline Actividades que implican esfuerzo & $\begin{array}{l}\text { Pulsar botones } \\
1 / 3 \text { del tiempo }\end{array}$ \\
\hline \multicolumn{2}{|l|}{ Factores de riesgo adicionales } \\
\hline Tarea de presición & Mas de la mitad del tiempo. \\
\hline Ritmo de trabajo & Ritmo totalmente determinado por la máquina \\
\hline \multicolumn{2}{|c|}{ Resultado final OCRA } \\
\hline \multicolumn{2}{|r|}{18,3} \\
\hline \multicolumn{2}{|r|}{ Resultado } \\
\hline & Riesgo medio \\
\hline
\end{tabular}

Nota: Los datos ingresados son el resultado de un pre - investigación de dos semanas. Se añadió una ayuda que disminuye el tiempo de exposición a la mitad. 


\section{Conclusiones}

La implementación de un programa de pausas activas resulta eficaz para la disminución de la fatiga, con la recomendación de implementar un programa permanente de pausas activas en la jornada laboral, con distintos tiempos, ejercicios y actividades que permitan al trabajador controlar la fatiga. Los resultados apoyan la idea de que las pausas activas producen cambios en el nivel de actividad física de las personas, puesto que se produjo un decremento del índice OCRA en tan solo 2 semanas de aplicación de las pausas activas, lo que nos lleva a pensar que, de llegarse a aplicar estas pausas por más tiempo, el índice inclusive podría disminuir aún más.

Al implementar pausas activas y dotar de ayuda de un asistente se redujo un $70 \%$ el nivel de riesgo ergonómico por movimiento repetitivo por el índice OCRA pasando de intolerable a medio.

\section{Referencias bibliográficas}

Alvaro, A., \& Ricardo, F. (2017). Evaluación del nivel de riesgo ergonómico por movimientos repetitivos en una línea de procesamiento de aves de corral aplicando los métodos art tool y ocra check list.

Buitrón Carrera, D. A. (2015). Estudio ergonómico sobre trastornos músculo esqueléticos por posturas forzadas en odontólogos en el Hospital de Especialidades de las Fuerzas Armadas $N^{\circ} 1$. Universidad Internacional SEK,

Cimatti, A., Dorigatti, M., \& Tonetta, S. (2013). OCRA: A tool for checking the refinement of temporal contracts. Paper presented at the 2013 28th IEEE/ACM International Conference on Automated Software Engineering (ASE).

Corbí-Santamaría, M., Baños-Martínez, V., Palomo, E. R., Llamazares, C. E., Rico, I. L., Cámara, C. P., . . . Psychology, E. (2014). Ergonomía y actividad física en mayores. 1(2), 243-252.

Cordero, A., Masiá, M. D., \& Galve, E. J. R. E. d. C. (2014). Ejercicio físico y salud. 67(9), 748-753.

Grieco, A. J. E. (1998). Application of the concise exposure index (OCRA) to tasks involving repetitive movements of the upper limbs in a variety of manufacturing industries: preliminary validations. 41(9), 1347-1356.

Işık, A. T., \& Adalı, E. A. J. I. J. o. A. O. M. (2016). A new integrated decision making approach based on SWARA and OCRA methods for the hotel selection problem. $8(2), 140-151$.

Laguerre, J. (2019). la Tendinitis laboral, riesgos ergonómicos en Odontología.

Martínez Barranco, M. P., \& Yandún Burbano, E. D. (2017). Seguridad y salud ocupacional en Ecuador: Contribución normativa a la responsabilidad social organizacional.

Niño Peña, J. E., \& Solano Aguado, M. (2020). Importancia de las Pausas Activas: Una Revisión Bibliográfica. Corporación Universitaria Minuto de Dios,

Picazo, A. R., \& de Miguel, J. L. (2003). NTP 629: Movimientos repetitivos: métodos de evaluación Método OCRA: actualización. 
Ribadeneira Campozano, M. S. (2015). Diseño de un programa de pausas activas para disminuir el riesgo ergonómico en el área de descabezado de Industrial Pesquera Santa Priscila SA. Universidad de Guayaquil. Facultad de Ingeniería Industrial. Maestría en ...,

Romero Moreno, F. S., \& Córdoba Quejada, H. (2021). Las pausas activas lúdico-recreativas como propuesta alternativa para la prevención del consumo del tabaco en los conductores del sistema integrado de transporte (SITP) de la calle 183 patio norte.

Rosero Menéndez, F. X. (2012). Evaluación de factores de riesgo ergonómico en una planta de producción de calzado. Quito, 2012.,

Soto, A. H., \& Casado, E. Á. J. G. p. d. r. 1. I. y. d. d. 1. g. d. 1. p. (2006). El método OCRA: evaluación del riesgo asociado al trabajo repetitivo de las extremidades superiores. (30), 28-33.

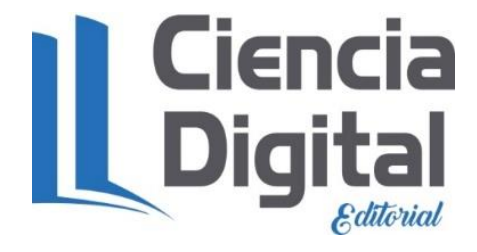


PARA CITAR EL ARTÍCULO INDEXADO

Pilco Albán, D. H., Córdova Suarez, M. A., \& Villacres Cevallos, E. P. (2021). Pausas activas para el control de trastornos músculo-esqueléticos en los odontólogos del seguro social campesino de la provincia de Cotopaxi. Anatomía Digital,4(3.1), 118-128. https://doi.org/10.33262/anatomiadigital.v4i3.1.1898

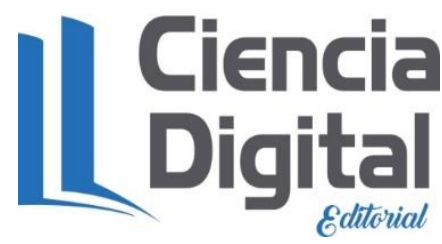

El artículo que se publica es de exclusiva responsabilidad de los autores y no necesariamente reflejan el pensamiento de la Revista Anatomía Digital.

El artículo queda en propiedad de la revista y, por tanto, su publicación parcial y/o total en otro medio tiene que ser autorizado por el director de la Revista Anatomía Digital.
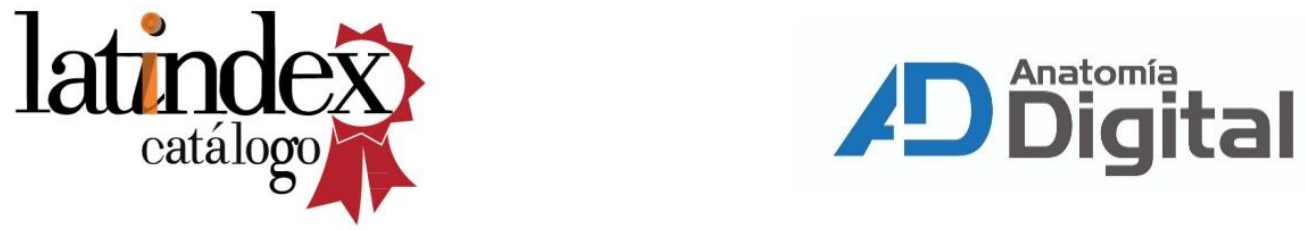